\title{
Role of Pyrroloquinoline Quinone in Biocontrol Together with Induced Systemic Resistance: A Novel Resource Trialed for Rice Disease Control
}

\author{
Muhammad Naveed ${ }^{1 *}$, Abdul Samad Mumtaz ${ }^{2}$ and Mushtaq Ahmad Saleem ${ }^{1}$ \\ ${ }^{1}$ Department of Biotechnology, Faculty of Life Sciences, University of Central Punjab, Lahore 54000, Pakistan \\ ${ }^{2}$ Department of Plant Sciences, Quaid-e-Azam University, Islamabad 44000, Pakistan \\ *For correspondence: naveed.quaidian@gmail.com; dr.naveed@ucp.edu.pk \\ Received 21 March 2020; Accepted 02 September 2020; Published 10 December 2020
}

\begin{abstract}
Plants show strong levels of resistance to an extensive range of pathogens on account of root colonization through plant growth-promoting rhizobacteria (PGPR), namely, induced systemic resistance (ISR). Little is known about bacterial determinants and plant signaling pathways that underpin ISR in cereal crops associated with ISR in dicotyledonous plants. The present study evaluates the potential of Pseudomonas spp. QAU-92 using site directed mutagenesis of the pqqC gene to elicit ISR in rice (Oryza sativa L.) against the fungal pathogen Cochliobolus miyabeanus. The comparison between the wild-type strain and the mutant strain for biochemical attributes, in vitro and in vivo antagonistic activity, carbon source utilization assay and in vivo analyses on rice (cv. C-039) revealed the statistically significant role of Pyrroloquinoline Quinone (PQQ) in plant growth promotion. RT-qPCR analysis revealed that the plant recognition of QAU-92 results in the activation of ethylene (ET) and jasmonic acid (JA) pathways and also shows clear differences in resistance against $C$. miyabeanus disease compared with the pqqC mutants (QAU92-2). The expression of TF 89 (EBP89), a susceptible gene, as well as the pathogenesis-related protein 1a $(P R l a)$ were much higher in the infected control and $p q q C$ mutant plant than in wild type inoculated plants. Hence, this study is the first of the kind that has investigated the expressional analysis of PQQ against antifungal activity, phosphate solubilization and the induced systemic resistance of QAU-92 against C. miyabeanus in rice. Additionally, PQQ genes may act as a key regulator of PRIa/ET cross-talk and its interference with the fungal manipulation of plants. (C) 2021 Friends Science Publishers
\end{abstract}

Keywords: Induced systemic resistance; Fungal pathogens; Pyrroloquinoline Quinone; expressional analysis; Oryza sativa; plant growth-promoting rhizobacteria

\section{Introduction}

Generally, plants have elaborate levels of inducible resistance that triggers pathogen infection and builds an initial line of defense/resistance (Adamowicz et al. 1991). Plant hormones such as ethylene (ET), abscisic acid, salicylic acid (SA) and jasmonic acid (JA) play pivotal role in these inducible responses, which are controlled by a network of interrelated signal transduction pathways (Adie et al. 2007; Robert-Seilaniantz et al. 2007; Asselbergh et al. 2008). Much of the recently reported evidences support the view that these signaling pathways are not independent and are influenced by a multifaceted network of antagonistic and synergistic interactions (Koornneef and Pieterse 2008). Such advanced interactions between defense pathways are assumed to give plants the regulatory potential to adapt their resistance response to complement an encounter with the attacker.

An investigation into the signal transduction pathways in activated plants suggests that some of the analogous pathways triggered by Bacillus spp. are similar to those activated by Pseudomonas spp. In some instances, during the activity of signal transduction pathways, the defense gene PRI accumulates in plants using ISR induced by Bacillus spp. (Kumar et al. 2012). Besides the effective resistance against an immunent pathogen attack, plants also achieve the development of resistance at the site of infection. The occurrence of this, which is called induced resistance, may be activated by a range of abiotic and biotic stimuli (Bostock 2005). Research in recent years has shown that the improved defensive ability of plants does not need to be triggered directly but may be due to the quicker and stronger expression of the basal defense response upon encountering a pathogen attack (Huot et al. 2014).

In the category of crops, rice (Oryza sativa L.) is an essential component of diet for more than three billion individuals in the tropical and sub-tropical Asia (Khush 2004) and is only rivaled by maize and wheat in importance. Yet, more than 70 diseases that inflict fungi, nematodes,

To cite this paper: Naveed M, AS Mumtaz, MA Saleem (2021). Role of pyrroloquinoline quinone in biocontrol together with induced systemic resistance: A novel resource trialed for rice disease control. Intl J Agric Biol 25:1-10 
viruses and/or bacteria on rice, which hamper its production, have been well documented. Of these, brown spot disease (Cochliobolus miyabeanus), sheath blight (Rhizoctonia solani) and rice blast (Magnaporthe oryzae) are the significant fungal constraints. Recently, brown spot disease in rice, which is triggered by $C$. miyabeanus, is a major disease in rain-fed ecosystems that badly affects yield and significantly diminishes rice quality (Paz et al. 2006). In recent years, sheath blight and rice blast diseases have been controlled by multiple $P G P R$ strains but there are little reports on brown spot. These include Pseudomonas fluorescens PF1 and FP7 (Nandakumar et al. 2001), P. fluorescens PfALR2 (Rabindran and Vidhyasekaran 1996), and Bacillus subtilis MBI 600 (Kumar et al. 2012), are effective against sheath blight; while the Pseudomonas strains, 7NSK2 and WCS374r, are effective against rice blast (Vleesschauwer et al. 2006). The root colonization of PGPR strains leads to form-induced resistance (discussed in terms of ISR) (Loon et al. 1998).

Only limited studies have reported the hormonal control or ISR-based analyses to control brown spot disease in rice. One of these was on Magnaporthe grisea KI-409 (a rice pathogen), the mutation of pqqA and pqqB genes in $E$. intermedium wiped out their bio-control capability. In addition to their capacity to improve the systemic resistance to infections activated by fungal pathogens, they advocated the involvement of PQQ in antifungal activity, as well as the embellishment of the systemic resistance and mineral phosphate solubilization of E. intermedium (Han et al. 2008). The PQQ biosynthesis pathway has been found to be comprised of six most conserved PQQ genes (pqqABCDEF) in most of the bacterial strains and pqqC (cofactor-less PQQ synthase) catalyzes the final step in PQQ biosynthesis pathway (Magnusson et al. 2004). According to review of the literature, very little attention has been paid on the expressional analysis of PQQ against pathogenic fungi in plants. Moreover, the bio-control potential of Pseudomonas spp. QAU-92 and its PQQ mutant (QAU92-2) in triggering ISR against brown spot disease in rice is yet to be tested. Therefore, the present study aimed to investigate the expression analysis of PQQ against antifungal activity; induced systemic resistance, phosphate solubilization of Pseudomonas spp. QAU-92 against $C$. miyabeanus; and finally, the extent of resistance against a $C$. miyabeanus attack on rice.

\section{Materials and Methods}

\section{Microorganism and culturing conditions}

The strain QAU-92 was isolated from wheat cultivar rhizospheres and cultured in $T Y$ medium at $28^{\circ} \mathrm{C}$ while the E. coli cultured in $\mathrm{LB}$ at $37^{\circ} \mathrm{C}$ was used for transformation and $S$. cerevisiae on $P D A$ medium at $30^{\circ} \mathrm{C}$ (Table 1). All cultures were preserved at $-80^{\circ} \mathrm{C}$ in $\mathrm{LB}$ medium supplemented with $40 \%$ glycerol.

\section{Identification of isolate and amplification of PQQ operon}

The genomic DNA extraction of bacteria was done by the CTAB method as described by Naveed et al. (2014a). The identification of the isolate was done with $16 \mathrm{~S}$ rRNA through PCR recipe according to Naveed et al. (2014b). PCR amplification of PQQ genes was done with freshly designed oligonucleotides using sequence information of Pseudomonas spp. QAU-92 (Table 1). PCR was carried out according to the procedure described by Naveed et al. (2015). The amplified PCR products were sequenced commercially from LGC genomics (Germany) and compared with already published sequences in NCBI GenBank databases. The sequences generated were submitted to NCBI Genbank and enlisted at end of the text.

\section{In vivo cloning and site-specific mutagenesis and Phosphate solubilization}

To induce mutation in the pqq operon, the pqq biosynthesis "pqqC" was targeted for site directed mutation using PCR based knock out method (Naveed et al. 2015) as described for QAU-92. The deletion plasmid of Pseudomonas spp. QAU-92 was constructed in the same way (Table 1) and PCR confirmed the deletion of PQQ gene. The wild type strain was used as a negative control and E. coli strain with the plasmid was used as positive control. The absence of pqqC loci in deletion mutants were further confirmed by in vitro phenotypic analyses such as phosphate solubilization and secondary metabolite production and in vivo carbon source utilization, catabolism of enzymes and plant growth promotion. An experiment was conducted to assess the phosphate solubilization capacity in wild and mutant strains, i.e. by halo zone formation on Pikovskaya agar medium and $\mathrm{pH}$ measurement in broth medium as described by Naveed et al. (2014b).

\section{PQQ mutant characterization}

PQQ mutants were further characterized for utilization of carbon and nitrogen sources and their role in regulation of enzymatic activity for oxidation-reduction and fermentation processes by API-20E kit (Bio Merieux, U.S.A.), embedded with 20 biochemical tests based on enzyme and pathway regulation to assess differences among wild and mutant strains. PQQ mutants were tested to identify the enzymes for which pqq act as a cofactor was based on carbon source utilization assay. We used $1 \%$ of each eight carbon sources (glucose, acetate, Na-citrate, Na-succinate, manitol, glycerol, ethanol and methanol) with M-9 nutritional media (Naveed et al. 2015). The LB culture of all strains at $10^{6}$ CFU was placed overnight into M9 media after taking OD at $620 \mathrm{~nm}$ then in 96 well plates in 12 replicates. After $24 \mathrm{~h}$ the differences in OD of wild and mutant type strains were observed. 


\section{PQQ role in plant growth promotion}

The seeds of Phaseolus vulgaris 'Prelude' (Belgium) which is a model plant and easy to grow were used for in vivo plant experiments and Rice $c v$. C-039 (Japonica), having advantage to grow easily. After culturing the bacteria on KB medium, the seed coat was removed. The surface was sterilized twice with $2 \% \mathrm{NaOCl}$ for 10 min (gently shaken to have better contact between seeds and $\mathrm{NaOCl}$ ). Place the seeds on moist filter paper and keep them in an incubator at $28^{\circ} \mathrm{C}$ for 5 days to allow germination and sterilized the saline solution, cylinders and potting soil ( 2 times). The bacterial suspensions were $\left(5 \times 10^{7} \mathrm{CFU} / \mathrm{g}\right.$ soil) mixed with sterile potting soil (Structural; Snebbout, Kaprijke, Belgium) and distributed soil into white boxes (12 seedlings/700 g soil/box). Soil was mixed with non-sterile distilled water to make it wet before bacterial application. After this the seeds were sown. $\mathrm{FeSO}_{4}$ and $\left(\mathrm{NH}_{4}\right)_{2} \mathrm{SO}_{4}(2: 1 \mathrm{~g} / \mathrm{L})$ were used as nutrients to fertilize $(250 \mathrm{~mL}$ solution/box) the plants.

The data on growth parameters (shoot length, shoot weight, leave area index, dry weight, total number of leaves and plant root) was documented on ten plants from three replicates by software package S.P.S.S. 15.0. The nonparametric data was analyzed using Kruskal-Wallis and Mann-Whitney comparisons $(\alpha=0.05)$.

\section{Biocontrol activity of Pseudomonas strains against Rhizoctonia root rot}

The bio-control activity Pseudomonas spp. QAU-92 and its derived pqqC mutant (Pseudomonas spp. QAU92-2) strains were tested in vitro for antifungal activity against Rhizoctina solani and Pythium spp. It was then re-confirmed in vivo with bean plants. The P. vulgaris 'Prelude' (Het Vlaams Zaadhuis, Belgium) was used to check the efficiency of Pseudomonas strain to suppress the Rhizoctonia root rot. Inoculum of $R$. solani (AG 2-2) was developed on watersoaked wheat seeds, which were then autoclaved two times on 2 successive days. The disease symptoms were recorded according to Nerey et al. (2010). All experiments were carried at $25^{\circ} \mathrm{C}$ with $16 \mathrm{~h}$ photoperiod and repeated with four replications per treatment having ten bean plants per replication along with infected and healthy controls.

\section{Root colonization by Pseudomonas spp. QAU92 and its PqqC mutant QAU92-2}

The actual rating of disease severity depends upon Pseudomonas spp. QAU-92 and mutant colonization with bean roots which were determined as mentioned by D'Aes et al. 2011). S.P.S.S. 15.0 software package was used for statistical analysis of data. Neither the data of root colonization experiments nor the ordinal data of the disease severity met the conditions of homogeneity and normality of variances. Therefore, the nonparametric Kruskal-Wallis and Mann-Whitney analyses $(\alpha=0.05)$ were executed.

\section{PQQ and Induced systemic resistance (ISR) in rice}

Two experiments i.e., rice plants (in vivo) and with rice cell suspension cultures (in vitro) were performed to assess the role of wild and $p q q C$ mutant strains in suppressing the rice brown spot disease through ISR.

\section{The analysis of cell lines treated with Pseudomonas supernatant by $q$ PCR}

The wild type Pseudomonas spp. QAU-92 strain and its pqqC mutant Pseudomonas spp. QAU92-2 strains culture was scraped off from LB medium plates and then put into the sterile $10 \mathrm{~mL}$ of demineralized water. The suspended bacterial colonies were centrifuged at $10,000 \mathrm{~g}$ for $10 \mathrm{~min} .1$ $\mathrm{mL}$ of supernatant was passed through a filter of $0.22 \mu \mathrm{m}$ and then added to $3 \mathrm{~mL}$ of 5-day-old rice cell. RNA extraction analysis was done from the cells collected at 1,3 and $6 \mathrm{~h}$ post inoculation (hpi). The LB broth was used as control. Following treatment with wild type Pseudomonas and $p q q C$ mutant strains, expression of JA marker genes $J A M Y B$ and JiOsPRIO were checked along with the ETrelated gene EBP89 and Actin (Os03g071810) used as an internal reference (Vleesschauwer et al. 2010) to normalize the gene expression levels.

\section{Induced resistance bio-assays and pathogen inoculations}

Induced resistance bio-assays for Pseudomonas spp. QAU92 and its pqqC mutant Pseudomonas spp. QAU92-2 were performed as described by Vleesschauwer et al. (2006) and Chandler et al. (2015) with some alteration. The $C$. miyabeanus strains Cm988 (brown spot) used for infection trials were grown on $P D A$ at $28^{\circ} \mathrm{C}$ for sporulation. Seven days-old mycelia were spread on the medium under blue light for three days to prompt sporulation. Conidia were harvested upon sporulation and to make a final density of $1 \times 10^{4}$ conidia $\mathrm{mL}^{-1}$ re-suspended in $0.5 \%$ gelatin (SigmaAldrich). For inoculation, 6.5-leaf stage of five-week-old seedlings was glazed over with conidial suspension $(1 \mathrm{~mL}$ per plant) by an artist airbrush. Straightway, plants were shifted to a precipitation chamber $\left(30^{\circ} \mathrm{C}\right.$, with humidity > $92 \%$ ) to assist fungal penetration and moved to greenhouse $\left(28^{\circ} \mathrm{C} \pm 4^{\circ} \mathrm{C}\right)$ after $18 \mathrm{~h}$ for development of disease. Leaf samples of infected, mock and control rice plants were collected after fungal inoculation at four-time points (i.e., 12 h, $24 \mathrm{~h}, 36 \mathrm{~h}$ and $48 \mathrm{~h}$ after inoculation) in two biological repeats. For two bacterial inocula Pseudomonas spp. QAU92 and its $p q q C$ mutant; a total of 80 samples of Pseudomonas spp. QAU92-2 were collected; 40 for each of the two biological repeats, RNA extraction and RT-PCR expression analyses.

\section{RNA extraction and cDNA synthesis}

After harvesting, rice leaf tissues were submerged in liquid 
nitrogen quickly to avoid possible RNA degradation. TRI reagent (Sigma) was used for total RNA extraction from frozen tissue and samples were quantified at A260/280 ratio (values should be < 1.6). After that it was re-suspended in $40 \mu \mathrm{L}$ of DEPC-treated RNase-free Milli Q and incubated for $10 \mathrm{~min}$ at $65^{\circ} \mathrm{C}$. The extracted RNA was treated with DNase (Turbo DNase, Applied Biosystems). The final concentration of extracted RNA was measured using Nanodrop ND-1000 Spectrophotometer. First cDNA strand was prepared by GoScript Reverse Transcription System (Promega, U.S.A.) from $1 \mu \mathrm{g}$ of RNA.

\section{Gene expression and quantitative real time PCR ( $q$ RT- PCR) analysis}

Quantitative RT-PCR ( $q$ RT-PCR) amplifications and gene expressional analysis were done as mentioned by Chandler et al. (2015). The plant RNA from all samples was standardized with internal control of actin (OsO3g0718100) or eukaryotic translation elongation factor 1A (eEFla Os03g0178000). The samples from control cell cultures; designated as a calibrator, pathogenesis-related (PR) class 1 (PRla) and Ethylene-responsive TF89 susceptible gene (EBP89) were used for $p q q$ gene expression analysis. Data was compiled by mean and standard error of three replicates from each representative experiment. Primer sequences listed in Table 5 were used for expressional analysis.

\section{Statistical analysis}

Analysis of variance (ANOVA) tests were used to analyze data at a confidence level of 95\%, together with KruskalWallis Test using Statistix 8.1 software (Tallahassee, USA) based on the method described by Steel et al. (1997).

\section{Results}

Identification based on the $16 \mathrm{~S}$ rRNA and rpoB sequences revealed that QAU-92 is a Pseudomonas spp.; however, it has low bootstrap support that is, 35 and $43 \%$, respectively (Fig. 1a and b). Furthermore, the Pseudomonas spp. QAU92 showed a capability to solubilize phosphate, which further demonstrated its impact on plant growth. On top of this, it produced the lipopeptides that boosted strain efficiency against plant diseases (Table 1).

\section{Amplification of the PQQ operon and characterization of the pqqC mutant}

The PCR amplification and sequence homology of the PQQ operon (pqqAB, pqqBCD, pqqE and pqqF) demonstrated more than $97 \%$ sequence similarity with the PQQ sequences of Pseudomonas already available in the NCBI GenBank Accession number CP003190. The pqqBCD was chosen to develop mutant strains that were identified through the detection of $1 \mathrm{~kb}$ segments. Therefore, QAU-92 produced

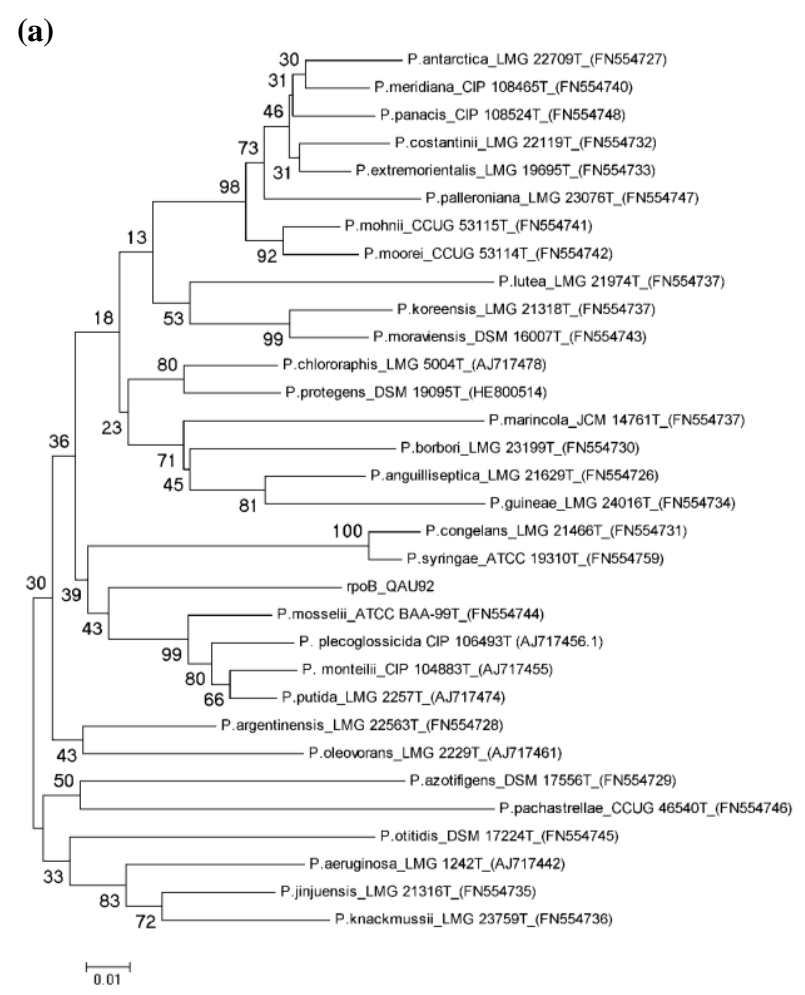

(b)

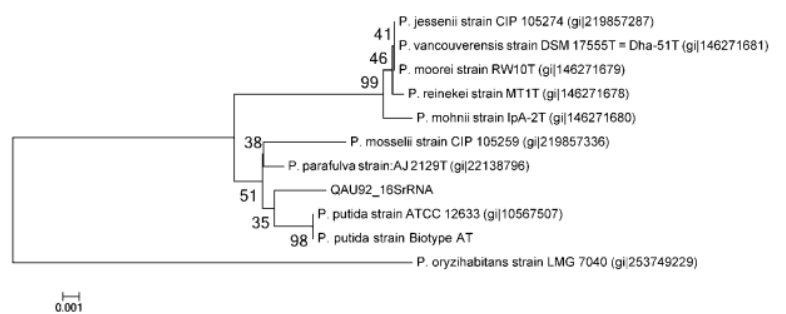

Fig. 1: Neighbor-joining phylogenetic tree showing (a) 16S rRNA and (b) rpoB gene sequence affinity of QAU-92 with Pseudomonas group. The $35 \%$ and $43 \%$ bootstrap value respectively provided statistical support base on 1000 interactions

eight mutants for the pqqC locus, and only the best characterized QAU92-2 was used here. The wild type and pqqC mutants were further confirmed and characterized for mutation and strain phenotype (Supp Fig. 1a, b and c).

The QAU-92 wild strain, tested for its capability to solubilize inorganic phosphate, demonstrated the utilization of ethanol as a carbon source. However, the pqqC mutant QAU92-2 was deficient in such activity, even in media enriched with ethanol (Table 2). Hence, the QAU-92 was an alcohol dehydrogenase (ADH) carrying system efficient in phosphate solubilization. The wild strain QAU-92 fermented various carbohydrates: L-omithin, glucose, sorbitol, Lrhamnose, D-melibiose, amygdaline and L-arabinose; while the mutant strain QAU92-2 lost this capacity (Table 2 ), suggesting the imminent role of PQQ in fermentation and redox reactions. 
The Role of PQQ in Biocontrol and ISR of Rice / Intl J Agric Biol, Vol 25, No 1, 2021

Table 1: Strains, plasmid vectors and primers used in this study

\begin{tabular}{|c|c|c|}
\hline $\begin{array}{l}\text { Strains/plasmid/ } \\
\text { Oligonucleotides }\end{array}$ & Characteristics and sequences $\left(5^{\prime} \rightarrow 3^{\prime}\right)$ & Reference/source \\
\hline Pseudomonas spp. QAU-92 & Biocontrol+, PGPR+, CLP+, pltC+, wild type (Pakistan) & This study \\
\hline Pseudomonas spp.QAU92-2 & Biocontrol-, PGPR-, CLP-, pqqC mutant (QAU92-2) & This study \\
\hline E. coli WM3064 & $\begin{array}{l}\text { Donor strain for conjugation; carries the pir gene, which is necessary for plasmids with an oriR6K origin } \\
\text { of replication }\end{array}$ & $\begin{array}{l}\text { Dietrich et al. } \\
\text { (2006) }\end{array}$ \\
\hline S. cerevisiae InvSc & Yeast strain for in vivo recombination (ura3-52/ura3-52 mutation) & Invitrogen \\
\hline $\begin{array}{l}\text { Rhizoctonia solani } \\
\text { AG 2-2 CuHav-Rs18 }\end{array}$ & Causal agent of root rot on bean (intermediately aggressive) (Cuba) & Nerey et al. (2010) \\
\hline $\begin{array}{l}\text { Plasmid } \\
\text { pMQ-30 } \\
\text { Oligonucleotides }\end{array}$ & Gene replacement vector for Pseudomonas spp.; sacB, URA3, GmR & Shanks et al. (2006) \\
\hline pqqC-Up-F & GGAATTGTGAGCGGATAACAATTTCACACAGGAAACAGCTGTTCAAGATGCTCAGCCACTG & Naveed et al. \\
\hline pqqC-Up-R & CAGTTCATAGGCCATGCTCAATGGGGATGTTCACCTGGTA & (2015) \\
\hline pqqC-Down-F & TACCAGGTGAACATCCCCATTGAGCATGGCCTATGAACTG & Naveed et al. \\
\hline pqqC-Down-R & CCAGGCAAATTCTGTTTTATCAGACCGCTTCTGCGTTCTGATCGATCTTGTCGATGTTGTGC & (2015) \\
\hline pqqBCD-F & TTCAAGATGCTCAGCCACTG & Naveed et al. \\
\hline pqqBCD-R & CGATCTTGTCGATGTTGTGC & (2015) \\
\hline PqqAB-F & TGTGGACCAAACCTGCATACACTG & Naveed et al. \\
\hline PqqAB-R & GATGCTCATGCCATCGAA & (2015) \\
\hline PqqE-F & GATCGTCCTCGCCTGAGTT & Naveed et al. \\
\hline PqqE-R & GATGACACGGGAGTTTCGAT & $(2015)$ \\
\hline PqqF-F & CCAACTTACCCTCGCCAAT & Naveed et al. \\
\hline PqqF-R & CAGCGTTGGCCAAACATAG & (2015) \\
\hline rpoB-F & CAGTTCATGGACCAGAACAACCCGCT & Naveed et al. \\
\hline rpoB-R & CCCATCAACGCACGGTTGGCGTC & (2015) \\
\hline
\end{tabular}

PGPR: plant growth promoting rhizobacteria; $\mathrm{PHZ}+$ : Phenazine producer; $\mathrm{CLP}+$ : lipopeptides production

Table 2: Biochemical characterization by API-20E kit, root colonization and in vitro antagonistic activity of Pseudomonas spp. QAU-92 and their derivatives pqqC mutant (QAU92-2) against $R$. solani $\mathrm{AG} 2-2$

\begin{tabular}{|c|c|c|}
\hline Tests & QAU-92 & QAU92-2 pqqC mutant \\
\hline Source (Rhizosphere) & wheat & wheat \\
\hline Strains identified by $16 \mathrm{~S}$ rRNA and $r p o \mathrm{~B}$ gene & Pseudomonas spp. & Pseudomonas spp. \\
\hline Strain group & Pseudomonas & Pseudomonas \\
\hline Drop collapse & drop collapse activity & no drop collapse activity \\
\hline Phosphate solubilization $^{\mathrm{a}}$ & $3.7 \pm 0.07$ & $0.5 \pm 0.03$ \\
\hline Utilization of Glucose $^{\mathrm{b}}$ & $0.4 \pm 0.02 \mathrm{a}$ & $0.2 \pm 0.03 b$ \\
\hline Utilization of ethanol & $1.2 \pm 0.04 b$ & $0.3 \pm 0.01 \mathrm{a}$ \\
\hline $\mathrm{GDH}^{\mathrm{c}}$ & $2250 \mathrm{bp}$ & $2250 \mathrm{bp}$ \\
\hline $\mathrm{PQQ}^{\mathrm{c}}$ & $4.9 \mathrm{~kb}$ & - \\
\hline Production of acid ${ }^{\mathrm{d}}$ & 4.14 & 5.92 \\
\hline Root colonization $^{\mathrm{e}}$ & $6.89 \pm 0.24 \mathrm{a}$ & $5.61 \pm 0.35 c$ \\
\hline Antagonistic $^{\mathrm{f}}$ & 2 & 0 \\
\hline Disease severity (DS) & $1.4 \pm 1.2$ & $3.2 \pm 1.2$ \\
\hline \multicolumn{3}{|c|}{${ }^{\mathrm{g}}$ Biochemical characterization (by API-20E kit) } \\
\hline SOR, RHA, SAC, MEL, AMY and ARY & + & - \\
\hline $\mathrm{ADH}$ and CIT & + & + \\
\hline LDC, H2S, URE, TDA, IND, VP, GEL and INO & - & - \\
\hline \multicolumn{3}{|c|}{ 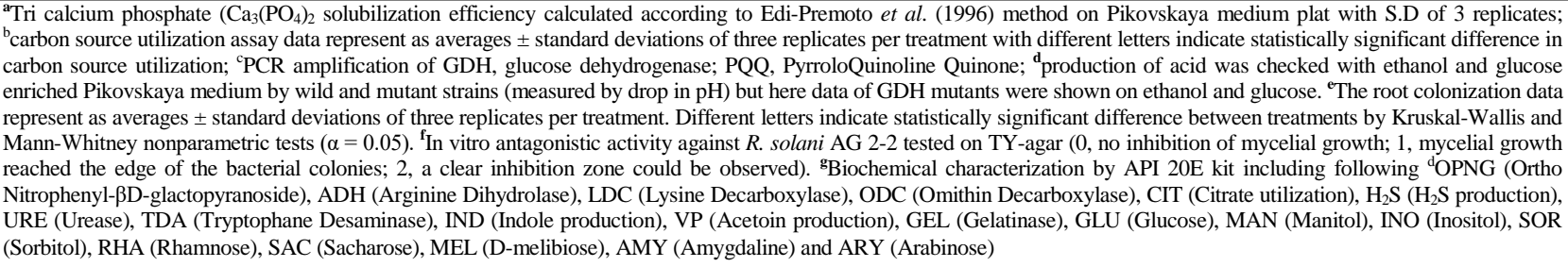 } \\
\hline
\end{tabular}

\section{Plant growth promotion activities in bean and rice}

The Kruskal-Wallis statistical data clearly demonstrated the behavior of the wild type (Pseudomonas spp. QAU-92) with the pqqC mutant (Pseudomonas spp. QAU92-2) strains, and the control inoculated plants based on height as well as the fresh weight of rice and bean. It was further confirmed through statistical analysis. The statistical analysis compared the performance of the wild type and mutants as they were assembled based on parameters such as plant height and fresh weight. In both cases, the $P$ value $=0.00<$ $0.05=\alpha$, rejected our null hypothesis (Table 4 ) and there was no difference between the performance of the wild type and mutants. Therefore, based on the test scores there existed enough evidences to conclude that there was difference among the three methods. Furthermore, the groups were 
Naveed et al. / Intl J Agric Biol, Vol 25, No 1, 2021

Table 3: Percent growth inhibition of phytophathogens by antagonistic Pseudomonas strains

\begin{tabular}{|c|c|c|c|c|c|c|}
\hline \multirow[t]{2}{*}{ Strains } & \multicolumn{2}{|c|}{ Rhizoctonia solani } & \multicolumn{2}{|c|}{ Fusarium solani } & \multicolumn{2}{|c|}{ Pythium spp. } \\
\hline & Mycelium growth $(\mathrm{mm})$ & Growth inhibition (\%) & Mycelium growth(mm) & Growth inhibition (\%) & Mycelium growth(mm) & Growth inhibition (\%) \\
\hline QAU-92 & $32.5 \mathrm{c}$ & $59.3 \mathrm{e}$ & $40 \mathrm{a}$ & $50 \mathrm{~g}$ & $37 \mathrm{~b}$ & $53.7 \mathrm{f}$ \\
\hline LSD, $0.5 \%$ & 0.876 & 0.864 & 0.867 & 0.865 & 0.842 & 0.867 \\
\hline
\end{tabular}

Table 4: Statistical analysis of wild type and pqqC mutant strains in plant growth promotion

\begin{tabular}{|c|c|c|c|c|c|c|c|}
\hline \multicolumn{2}{|c|}{ Wild and pqqC mutated Statistical } & \multicolumn{4}{|c|}{ Inoculation with Bean plants } & \multicolumn{2}{|c|}{ Inoculation with rice } \\
\hline Strains & parameters $^{\mathrm{a}, \mathrm{b}}$ & Plant height & Root length & Fresh weight $(\mathrm{S} \times \mathrm{R})$ & Leaf area $(\mathrm{L} \times \mathrm{W})$ & Plant Height* & Fresh weight* $(\mathrm{S} \times \mathrm{R})$ \\
\hline \multirow[t]{3}{*}{ QAU-92 and QAU92-2 } & Chi-Square & 14.296 & 14.296 & 14.318 & 14.296 & 25.812 & 21.862 \\
\hline & Df & 1 & 1 & 1 & 1 & 2 & 2 \\
\hline & Asymp. Sig. & 0.000 & 0.000 & 0.000 & 0.000 & 0.000 & 0.000 \\
\hline
\end{tabular}

Asymp. Sig (Asymptotic significance) = P-value, Df (Degree of freedom), a. Kruskal Wallis Test, b. Grouping Variable: CLASSES, L (Length), W (Width), S (Shoot), R (Root) and $*$ control treatment along with wild and mutant strains.

Table 5: Gene-specific primers for quantitative real-time PCR (qPCR)

\begin{tabular}{llllll}
\hline Pathway & Gene & Annotation & Locus number & Forward (5'-3') & Reverse (3'-5') \\
\hline Housekeeping & Actin & Rice actin 1 & Os03g0718100 & GCGTGGACAAAGTTTCAACCG & TCTGGTACCCTCATCAGGCATC \\
gene & eEF1a & Eukaryotic elongation factor 1A & Os03g0178000 & GGCTGTTGGCGTCATCAAGA & CCGTGCACAAAACTACCATT \\
Ethylene (ET) & EBP89 & Ethylene responsive TF 89 & Os03g0182800 & TGACGATCTTGCTGAACTGAA & CAATCCCACAAACTTTACACA \\
Jasmonic acid & JAMYB & JA-inducible Myb TF & Os11g0684000 & TGGCGAAACGATGGAGATGG & CCTCGCCGTGATCAGAGATG \\
(JA) & JiOsPR10 & JA-inducible PR10 protein & Os03g0300400 & CGGACGCTTACAACTAAATCG & AAACAAAACCATTCTCCGACAG \\
& OsPRla & $\begin{array}{l}\text { pathogenesis-related protein (PR) } \\
\text { class 1 }\end{array}$ & Os07g03710 & GTCGGAGAAGCAGTGGTACG & GGCGAGTAGTTGCAGGTGAT \\
& & & & & \\
\hline
\end{tabular}

tested and the median scores were equal. However, $\mathrm{p}=0.00$ $<0.05=\alpha$ provided reasons to reject the null hypothesis. The statistical analyses clearly showed and corresponded to the plant growth promotions.

\section{Assessing the bio-control capacity of Pseudomonas spp. QAU-92}

In vitro antagonistic activity: The Pseudomonas spp. QAU-92 strain significantly inhibited the growth of $R$. solani (59.3f) with 0.864 of least significant difference (LSD $\leq 0.05$ ). Furthermore, it showed an almost equal inhibition capability against Pythium spp. and Fusarium solani with 0.867 and 0.865 of least significant difference values, respectively. The statistical analysis further confirmed the bio-control potential of the Pseudomonas spp. QAU-92 strain. The results indicated it is a potential candidate for disease control in plants (Table 3).

In vivo antagonistic activity: The in vivo bio-control activity of Pseudomonas spp. QAU-92 against Rhizoctonia root rot revealed a substantial decrease in disease severity triggered by $R$. solani on bean plants. In contrast, the $p q q C$ mutant showed only a reduced potential in disease control. In another experiment, Pseudomonas spp. QAU-92 protected against a moderately aggressive isolate of $R$. solani AG 2-2 and caused a reduction in disease severity (DS): from $\approx 4.0 \pm 0.9$ to $1.4 \pm 1.2(p=0.000)$. Treatments with mutant strains showed less disease control activity; from $\approx 4.0 \pm 0.9$ to $3.2 \pm 1.2(p=0.000)$. The QAU92-2 mutant (deficient in PQQ production) demonstrated a complete loss in bio-control capacity and the DS for this strain was very near to that of the control (Table 2). This datum was also valid for a second trial, which yielded similar results. The count of the wild type (Pseudomonas spp. QAU-92) and the pqqC mutant strain (Pseudomonas spp. QAU92-2) showed bacterial population differences on the bean roots (Table 2). The concentrations of the wild and mutant bacterial strains were observed to be variable between repetitions and treatments of time. However, the concentration factor did not affect the disease-suppressive ability and revealed that the root colonization was appropriately higher for optimal biological control. For the most part, the mutant QAU92-2 lacking $p q q$ had the lowest bacterial root concentration and root colonization.

\section{Induced systemic resistance in rice}

The JA/ET signaling pathways: Following the treatment with Pseudomonas spp. QAU-92 and the Pseudomonas spp. QAU92-2 mutant supernatant, the hormone signaling pathways were observed. In addition to this, the expression levels of the JA marker genes; JiOsPRIO and JAMYB were recorded. 4- and 2-fold increase in the expression of genes respectively, was observed in infected plants. A 5- and 5fold increase was recorded in the $p q q C$ mutant (Pseudomonas spp. QAU92-2) inoculated plants compared with the wild type (Pseudomonas spp. QAU92), which had a higher expression of approximately 13- and 9-fold at 3 hpi, respectively (Fig. 2a and b). Under similar conditions, the ET-related gene EBP89 showed an a6-fold expression by the infected control plants, a 9-fold expression by the pqqC mutant (Pseudomonas spp. QAU92-2) inoculated plants, and an upregulation of wild type (Pseudomonas spp. QAU-92), expressed about 20-fold at 6 hpi (Fig. 2c). 


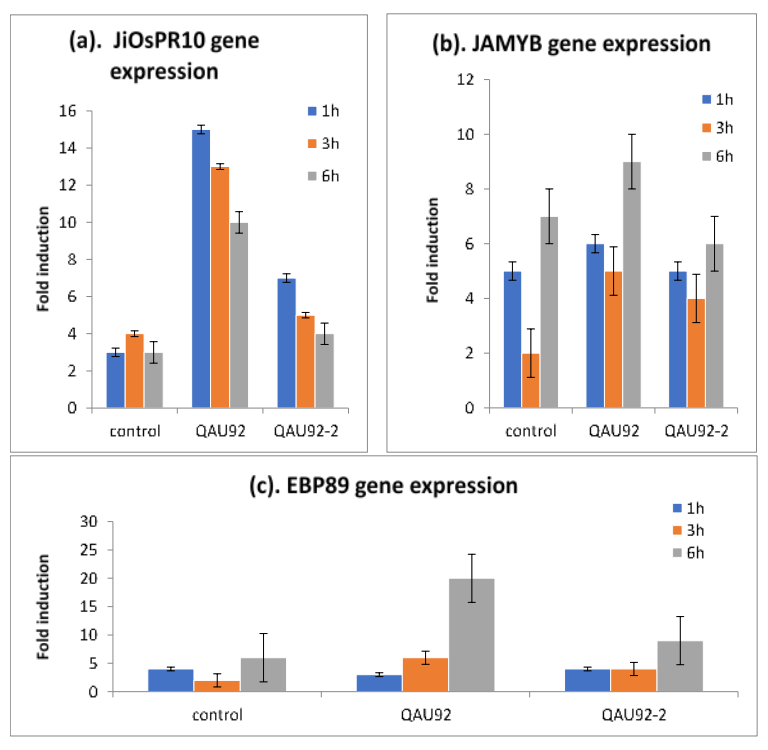

Fig. 2: Expression of hormone marker genes in rice cell cultures treated with supernatant of Pseudomonas spp. QAU-92 and its pqqC mutant (Pseudomonas spp. QAU92-2). At different time points after inoculation (1, 3 and $6 \mathrm{~h}$ ), cell cultures were harvested and subjected to quantitative RT-PCR analysis for the following transcripts: (a) JiOsPR10, (b) JAMYB and (c) EBP89. Actin (Os03g071810) was used as an internal reference to normalize the gene expression levels and calculated relative to the expression in mock-treated control cells at 1, 3 and $6 \mathrm{~h}$. Data presented are means and standard error of three replicates from a representative experiment

Together, these results suggested that $p q q$ genes produced activation of JA and ET pathways, while the $p q q$ deletion mutant was unable to activate the hormone to the level of the wild type Pseudomonas spp. QAU-92 strain.

Similarly, the quantitative reverse transcription analysis showed an accumulation of JA transcripts upon treatment with the Pseudomonas spp. QAU-92 supernatant (Fig. 2a and b) but not as much as with the pqqC mutant Pseudomonas spp. QAU92-2 supernatant. The application of the QAU-92 supernatant caused a strong and fast accumulation of JiOsPR10, with mRNA levels topping 1 hpi, and as 3-fold was found in mock-inoculated controls, 7fold in the $p q q C$ mutant (QAU92-2) and 15-fold in the upregulated with wild type (QAU92).

The use of the QAU-92 supernatant also induced a strong 11-fold upregulation of EBP89 (Fig. 2c) gene expression in comparison to the control and $p q q C$ mutant strain, while much weaker changes were observed in response to EBP89 at 1 and 3 hpi than with the mock control. These changes indicated that the PQQ produced in the LB broth-culture by the strain QAU-92 triggered the JA/ET signaling pathways.

\section{ISR against Cochliobolus miyabeanus in rice}

The PQQ-based induced resistance in rice (Oryza sativa subsp. indica $c v$ CO39) was assessed against the $C$. miyabeanus strain, $\mathrm{Cm} 988$, using the wild type (Pseudomonas spp. QAU-92) and its pqqC mutant (Pseudomonas spp. QAU92-2). The analysis showed clear differences in resistance against $C$. miyabeanus disease induced by wild type strains compared with the $p q q C$ mutants. Gene expression in the mock control, control and bacteria-treated samples were articulated as a ratio to actin or $e E F l a$ expression through measured efficiency for each gene by RT-PCR. Based on the expression of reference genes (actin and eEFla), the CT (cycle threshold) value of Eefla fluctuated more than did the actin gene and therefore actin was chosen as the reference gene.

\section{Expression analysis}

Ethylene-responsive TF 89 (EBP89), a susceptible gene used as a reference, was upregulated more in infected control plants than in the mock treatment, suggesting the infection-prone nature of plants. It was further observed that the EBP89 manifested a 21- and 18-fold higher expression in infected control plants than in mock control plants after 36 and 48 h post-inoculation (hpi), respectively (Fig. 3b). For both the wild type strains and pqq mutant strains, it showed less susceptibility to pathogens than did the control plants, which demonstrated resistance to the pathogen. All mutants showed greater susceptibility to pathogens than did wild type strains. The results of disease susceptibility in two biological repeats were the same, but the expression was much higher in the second biological repeat (Fig. 3a and b).

The EBP89 expression responded strongly to pathogen infection in the infected control plants and $p q q C$ mutants than it did in both wild types. This resulted in the expression of approximately 10 -fold by the infected control plants and 5-fold by the $p q q C$ mutant (Pseudomonas spp. QAU92-2) plants compared to the wild type (Pseudomonas spp. QAU92). The expression of the wild type (Pseudomonas spp. QAU-92) was only 0.26 -fold at $36 \mathrm{hpi}$ and 19-fold induction by the infected control plants. Furthermore, 13fold induction by the pqqC mutant (Pseudomonas spp. QAU92-2) was compared to the wild type (Pseudomonas spp. QAU-92), which induced only 6-fold at $48 \mathrm{hpi}$ in the second biological repeat (Fig. 3a). In conclusion, the suppression of Cm988-induced EBP89 expression consequently from the wild type (Pseudomonas spp. QAU92) plants was higher than the $p q q C$ mutant (Pseudomonas spp. QAU92-2) in two biological repeats. The results revealed that the pqq-induced resistance against $C$. miyabeanus in the wild type inoculated plants was higher than in the pqq deleted mutant plants.

\section{Pathogenesis-related protein (PR1a) expression analysis}

The expression of PR proteins is generally pathogen- and host-specific. The expression of the PR1a gene was much higher in the infected control and $p q q C$ mutant plant than in 
the wild type inoculated plants, resulting in the wild-type (Pseudomonas spp. QAU-92) plants exhibiting a higher induced systemic resistance than the $p q q C$ mutant (Pseudomonas spp. QAU92-2) in two biological repeats at all observation points (12 h, $24 \mathrm{~h}, 36 \mathrm{~h}$ and $48 \mathrm{~h}$ hpi) (Fig. 3b). Approximately, a 47-fold induction was observed by infected control plants and a 39-fold induction by $p q q C$ mutant (Pseudomonas spp. QAU92-2) inoculated plants. In contrast, the wild type (Pseudomonas spp. QAU92) induced about a 15 -fold at $36 \mathrm{hpi}$, a 46 -fold induction by infected control plants and a 37 -fold induction by the $p q q C$ mutant (Pseudomonas spp. QAU92-2). Also, the wild type (Pseudomonas spp. QAU-92) showed a 17-fold induction at 48 hpi in the second biological repeat (Fig. 3b). Hence, PQQ-induced resistance against $C$. miyabeanus in the wild type inoculated plants was recorded higher compared with the pqq deleted mutant plants. The OsPRla gene selected here clearly upregulated in a compatible $C$. miyabeanus fungus interaction, indicating that the $p q q$ gene has an effect in suppressing the disease.

\section{Discussion}

The identification of the microbes under study remains a fundamental yet tricky task and is usually done through amplifying conserved loci. The molecular phylogeny constructed, therefore, extends our knowledge with regard to organismic relationship and provides the basis for accurate identification (Singh et al. 2007). One of the most widely used loci has been the 16S rRNA gene sequences, and the rationale behind this is that the bacterial strains with a similarity of less than $97 \%$ can be stated as novel-although after complete characterization. In a similar study, the QAU-68 and QAU-63 strains showed 95 and 97\% 16S rRNA sequence similarity, and may be regarded as novel by further characterization (Naveed et al. 2014b). In the present study, the 16S rRNA sequence homology for some strains was found low compared with the already submitted sequences at NCBI. The QAU-92 isolates showed $89 \%$ homology with Pseudomonas putida, a level much lower than the threshold, which therefore pointed to its possible novel status. The housekeeping gene/MLSA analysis revealed that the QAU-92 strain also showed low-level homology and a poor bootstrap value $(43 \%)$ with loci used in MLSA (rpoB). The results therefore endorsed its novel nature. However, it is important to mention that its novel status requires complete taxonomic characterization (Lim et al. 2006), which is in progress.

Although PQQ has been reported in several bacterial genera, many of the species are living in an anaerobic environment and do not use glucose as a carbon source. These bacteria do not have a PQQ cofactor alone but have it in the form of PQQ-dependent GDH. The role of PQQ as cofactor is so important that the GDH enzyme remains inactive. The majority of Pseudomonas species (such as $P$. fluorescens) are strictly aerobic in nature and act as a glucose oxidizer (Choi et al. 2008). Such bacterial strains produce PQQ-dependent GDH and thus PQQ remains active in such cases. The API-20E system has been found appropriate for the identification of Gram-negative rods and enteric bacteria. The API-20E system for the biochemical characterization of the $p q q C$ mutant and wild type strains (QAU92) was used to check the effectiveness of the pqqC gene. Their role was deciphered through comparing the wild type and $p q q C$ mutants in the biochemical utilization of carbon, nitrogen sources and enzymatic action, which demonstrated that PQQ has an effect on processes like fermentation and oxidation-reduction (Table 2). These analyses highlighted the clear difference in phenotype of QAU92 wild and mutant strain.

To an extent, PQQ's role is connected to the uptake of phosphate via plants as a cofactor for rhizobacteria dehydrogenases. The PQQ assists in making the soil and the surrounding environment acidic (Rodriguez et al. 2004) and consequently more phosphate becomes available to plants. After the $p q q C$ deletion, the mutant strains (Pseudomonas spp. QAU92-2) lost their capability to solubilize phosphate in vitro and also their ability to acidify the medium. The present study confirmed that the phosphate solubilization activity and plant growth promotion is stopped after mutation in the pqq gene when compared with the wild type strain phenotype. Previously, it was reported that PQQ enhanced pollen germination in vitro in certain plant species such as, Tulipa, Lilium, Camellia, lettuce, bean and tomato (Naveed et al. 2015), but the mechanism remains unclear. The present study provided evidence that PQQ is a plant growth-promoting factor, which was demonstrated through comparison of such activity in Pseudomonas wild type strains and the loss of such activity in the $p q q C$ mutants (both in vitro with lettuce as well as in vivo in bean and rice). A significant difference $(P<0.05)$ was noted for plant height, shoot length, dry weight, root weight and total number of leaves in the wild type strains with the $p q q C$ mutants, and has shown that the plant growth promotion is mediated by PQQ. It is anticipated that this will enrich our present understanding of the plant growth promotion mechanism (Naveed et al. 2016). The PQQ synthesized from P. fluorescens B16 has been reported as a growth promoter in tomato, cucumber, Arabidopsis and hot pepper (Choi et al. 2008).

The Pseudomonas spp. QAU-92 produced biosurfactant (lipopeptides) also demonstrated biological control of $R$. solani root rot. Previously, Perneel et al. (2007) reported that $P$. fluorescens CMR12a produced phenazines and bio-surfactants in cocoyam root rot, suggesting strong antagonistic activity against $R$. solani. The reduced antifungal capacity and plant growth promotion might be due to low acid production by the $p q q C$ mutants compared with the wild type strains and further suggested the possible control of PQQ over such a process.

The pBKminiTn7- gfp2 tagging system revealed successful root colonization in lettuce by the Pseudomonas QAU-92, which colonized the root hair zone of the plant. 
This clearly indicated the expression of this gene in lettuce roots. This system has been found useful in environmental studies, disease control, and in addressing the gene expression and population dynamics in a plant's rhizosphere (Koch et al. 2001). The previously reported failures in plant growth promotion studies under field conditions have often been associated with poor root colonization (Bloemberg and Lugtenberg 2001). We found in the present study that the plant growth promotion by $p q q C$ mutants (QAU92-2) maintained the capability to colonize roots, which emphasized that many other factors might be involved in plant growth promotion beyond root colonization. This recognized a new PGPR factor PQQ from Pseudomonas spp. QAU-92, which also produced lipopeptides. The root colonization may be influenced by the production of the lipopeptides of both strains because the rhizosphere competence of the bio-surfactant was increased (D'Aes $e t$ al. 2011).

Many recent studies have accredited the importance of lipopeptides for bacterial root colonization and motility, which are often crucial aspects of biocontrol agents for the soil borne pathogens (Andersen et al. 2003; Tran et al. 2007). Very few studies are available on plant signaling pathways and bacterial factors underlying ISR in key cereal crops like rice, although the data on dicots is relatively high. The present study focused on PQQ genes of the QAU-92 strain producing a cyclic lipopeptides (CLP) type biosurfactant, and showed antagonistic activity against fungus and their role in increasing ISR in the model monocot rice. We observed that the application of Pseudomonas QAU-92 protected the foliar tissues of rice against brown spot diseases compared with the pqqC mutant strain QAU92-2, which showed a clear symptom of the disease on the leaves. We also identified pqq in Pseudomonas with ISR elicitingactivity (Fig. 2) and how it triggers the activation of SA and JA pathways but represses ET signaling, showing the presence of multiple ISR resistance pathways in rice (Fig. 2-3).

The first line of plant defense becomes activated upon pathogen recognition, which results in a basal level of resistance (Pieterse et al. 2009). When PGPR colonizes the host roots, it leads to ISR (Loon et al. 1998). The expression of the pathogenesis-related $(P R)$ protein is generally pathogenic and host-specific. In rice, it has shown infection with $C$. miyabeanus, inducing the transient expression of PRla. An approximately 47-fold induction by infected control plants, and a 39-fold induction by pqqC mutant (Pseudomonas spp. QAU92-2) inoculated plants as the wild type (Pseudomonas spp. QAU-92) induced approximately 15-fold at 36 hpi (Fig. 3b). Therefore, the PQQ induced resistance against $C$. miyabeanus in the wild type inoculated plants was recorded as higher compared with the $p q q$ deleted mutant plants. The OsPRla gene selected here clearly upregulated in a compatible $C$. miyabeanus fungus interaction, indicating that the $p q q$ gene has an effect in suppressing the disease.
Studies in rice and Arabidopsis have revealed that rhizobacterial-mediated ISR functions have components of ET and JA response pathways and are independent on SA (Verhagen et al. 2004). The expression of the JA marker genes JiOsPRIO and JAMYB responded strongly to the QAU-92 treatment compared with the $p q q C$ mutant (QAU92-2). At the same time point and for the same treatment, the ET-related gene EBP89 showed upregulation over the mock-treated controls and $p q q C$ mutant (Fig. 3a). These results also suggested that $p q q$ genes of QAU-92 in LB broth produced activation of mainly JA and ET pathways, while the $p q q$ deletion mutant (QAU92-2) was unable to activate the hormone up to the level of wild type $P$. fluorescens QAU-92. A clear difference between the wild type (Pseudomonas spp. QAU92) and the pqqC mutant (Pseudomonas spp. QAU92-2) strains in the expression of an ET-related gene EBP89 and the JA marker genes $J i O s P R 10 P R l a$ gene and JAMYB showed the fundamental role of PQQ in induced systemic resistance against $C$. miyabeanus in rice and against $R$. solani root rot in bean.

\section{Conclusion}

This is the first study that has investigated the expressional analysis of PQQ from Pseudomonas spp. QAU-92 against antifungal activity, phosphate solubilization and the induced systemic resistance against $C$. miyabeanus in rice. Furthermore, evaluated the extent of resistance against a $C$. miyabeanus attack on rice

\section{GenBank sequence submissions}

The EMBL GenBank accession numbers for the 16S rRNA gene sequence of QAU-92 strain is KM251450 and rpoB gene sequences is KM251446. The PQQ operon of QAU-92 are pqqA (KM251432), pqqB (KM251433), pqqC (KM251434), pqqD (KM251435) and pqqE (KM251436) and glucose dehydrogenase ( $\mathrm{gdh}$ ) encoding gene sequence is $\mathrm{KM} 251439$.

\section{Acknowledgements}

The financial supports (SRGP) provided by Higher Education Commission (HEC) of Pakistan and Phytopathology Lab, University of Ghent, Belgium for $\mathrm{Ph}$.D. research work of a scholar of this faculty are highly appreciated.

\section{Author Contributions}

$\mathrm{MN}$ performed core research work in this article which includes role of Pyrroloquinoline Quinone (PQQ) in biocontrol and induced systemic resistance in rice disease control and expressional analysis of PQQ against phosphate solubilization, antifungal activity. ASM supervised this work, provided facilitates for bench work and generously 
made available the chemicals, materials, and equipment this research work. MAS improved the quality of manuscript and did the proof reading.

\section{References}

Adamowicz M, T Conway, KW Nickerson (1991). Nutritional complementation of oxidative glucose metabolism in Escherichia coli via pyrroloquinoline quinone-dependent glucose dehydrogenase and the Entner-Doudoroff pathway. Appl Environ Microbiol 57:2012-2015

Adie B, J Chico, I Rubio-Somoza, R Solano (2007). Modulation of plant defenses by ethylene. J Plant Growth Regul 26:160-177

Andersen JB, B Koch, TH Nielsen, D Sørensen, M Hansen, O Nybroe, C Christophersen, J Sørensen, S Molin, M Givskov (2003). Surface motility in Pseudomonas spp. DSS73 is required for efficient biological containment of the root-pathogenic microfungi Rhizoctonia solani and Pythium ultimum. Microbiology 149:37-46

Asselbergh B, DD Vleesschauwer, M Höfte (2008). Global switches and fine-tuning-ABA modulates plant pathogen defense. Mol PlantMicrob Interact 21:709-719

Bloemberg GV, BJJ Lugtenberg (2001). Molecular basis of plant growth promotion and biocontrol by rhizobacteria. Curr Opin Plant Biol 4:343-350

Bostock RM (2005). Signal crosstalk and induced resistance: straddling the line between cost and benefit. Annu Rev Phytopathol 43:545-580

Chandler S, NV Hese, F Coutte, P Jacques, M Höfte, DD Vleesschauwer (2015). Role of cyclic lipopeptides produced by Bacillus subtilis in mounting induced immunity in rice (Oryza sativa L.). Physiol Mol Plant Pathol 91:20-30

Choi O, J Kim, JG Kim, Y Jeong, JS Moon, CS Park, I Hwang (2008). Pyrroloquinoline quinone is a plant growth promotion factor produced by Pseudomonas fluorescens B16. Plant Physiol 146:657-668

D'Aes J, GKH Hua, KD Maeyer, J Pannecoucque, I Forrez, M Ongena, LEP Dietrich, LS Thomashow, DV Mavrodi, M Höfte (2011). Biological control of Rhizoctonia root rot on bean by phenazine- and cyclic lipopeptide-producing Pseudomonas CMR12a. Phytopathology 101:996-1004

Dietrich LE, A Price-Whelan, A Petersen, M Whiteley, DK Newman (2006). The phenazine pyocyanin is a terminal signalling factor in the quorum sensing network of Pseudomonas aeruginosa. Mol Microbiol 61:1308-1321

Edi-Premoto M, AM Moawad, PLG Vlek (1996). Effect of phosphate solubilizing Pseudomonas putida on the growth of maize and its survival in the rhizosphere. Indones J Crop Sci 11:13-23

Han SH, CH Kim, JH Lee, JY Park, SM Cho, SK Park, KY Kim, HB Krishnan, YC Kim (2008). Inactivation of pqq genes of Enterobacter intermedium $60-2 \mathrm{G}$ reduces antifungal activity and induction of systemic resistance. FEMS Microbiol Lett 282:140-146

Huot B, J Yao, BL Montgomery, SY He (2014). Growth-defense tradeoffs in plants: A balancing act to optimize fitness. Mol Plant 7:1267-1287

Khush GS (2004). Rice Genetics IV, pp:439-447. International Rice Research Institute, Los Baños, Philippines

Koch B, LE Jensen, O Nybroe (2001). A panel of Tn7-based vectors for insertion of the gfp marker gene or for delivery of cloned DNA into Gram-negative bacteria at a neutral chromosomal site. J Microbiol Meth 45:187-195

Koornneef A, CMJ Pieterse (2008). Cross talk in defense signaling. Plant Physiol 146:839-844

Kumar KVK, SKR Yellareddygari, MS Reddy, JW Kloepper, KS Lawrence, XG Zhou, H Sudini, DE Groth, KS Raju, ME Miller (2012). Efficacy of Bacillus subtilis MBI 600 against sheath blight caused by Rhizoctonia solani and on growth and yield of rice. Rice Sci 19:55-63

Lim JM, CO Jeon, JC Lee, YJ Ju, DJ Park, CJ Kim (2006). Bacillus koreensis spp. nov., a spore-forming bacterium, isolated from the rhizosphere of willow roots in Korea. Intl J Syst Evol Microbiol 56:59-63

Loon LCV, PAHM Bakker, CMJ Pierterse (1998). Systemic resistance induced by rhizosphere bacteria. Annu Rev Phytopathol 36:453-483
Magnusson OT, H Toyama, M Saeki, R Schwarzenbacher, JP Klinman (2004). The structure of a biosynthetic intermediate of pyrroloquinoline quinone (PQQ) and elucidation of the final step of PQQ biosynthesis. J Amer Chem Soc 126:5342-5343

Nandakumar R, S Babu, R Viswanathan, T Raguchander, R Samiyappan (2001). Induction of systemic resistance in rice against sheath blight disease by Pseudomonas fluorescens. Soil Biol Biochem 33:603-612

Naveed M, T Komal, S Haleema, A Haroon, MA Samad (2016). The life history of Pyrroloquinoline quinone (PQQ): A versatile molecule with novel impacts on living systems. Intl J Mol Biol Open Access 1:29-46

Naveed M, Y Sohail, N Khalid, I Ahmed, AS Mumtaz (2015). Evaluation of glucose dehydrogenase and pyrroloquinoline quinine (pqq) mutagenesis that renders functional inadequacies in host plants. $J$ Microbiol Biotechnol 25:1349-1360

Naveed M, I Ahmed, N Khalid, AS Mumtaz (2014a). Bioinformatics based structural characterization of glucose dehydrogenase (gdh) gene and growth promoting activity of Leclercia spp. QAU-66. Braz J Microbiol 45:603-611

Naveed M, S Mubeen, S Khan, I Ahmed, N Khalid, HAR Suleria, A Bano, AS Mumtaz (2014b). Identification and characterization of rhizospheric microbial diversity by $16 \mathrm{~S}$ ribosomal RNA gene sequencing. Braz J Microbiol 45:985-993

Nerey Y, J Pannecoucque, HP Hernandez, M Diaz, R Espinosa, SD Vos, SV Beneden, L Herrera, M Höfte (2010). Rhizoctonia spp. causing root and hypocotyl rot in Phaseolus vulgaris in Cuba. J Phytopathol $158: 236-243$

Paz MAGD, PH Goodwin, AK Raymundo, EY Ardales, CMV Cruz (2006). Phylogenetic analysis based on ITS sequences and conditions affecting the type of conidial germination of Bipolaris oryzae. Plant Pathol 55:756-765

Perneel M, J Heyrman, A Adiobo, KD Maeyer, JM Raaijmakers, PD Vos, M Höfte (2007). Characterization of CMR5c and CMR12a, novel fluorescent Pseudomonas strains from the cocoyam rhizosphere with biocontrol activity. J Appl Microbiol 103:1007-1020

Pieterse CMJ, A Leon-Reyes, SVD Ent, SCMV Wees (2009). Networking by small-moleculehormones in plantimmunity. Nat Chem Biol 5:308-316

Rabindran R, P Vidhyasekaran (1996). Development of a formulation of Pseudomonas fluorescens PfALR2 for management of rice sheath blight. Crop Prot 15:715-721

Robert-Seilaniantz A, L Navarro, R Bari, JDG Jones (2007). Pathological hormone imbalances. Curr Opin Plant Biol 10:372-379

RodriguezH, TGonzalez, I Goire, Y Bashan (2004). Gluconic acid production and phosphate solubilization by the plant growth-promoting bacterium Azospirillum spp. Naturwissenschaften 91:552-555

Shanks RM, NC Caiazza, SM Hinsa, CM Toutain, GA O'Toole (2006). Saccharomyces cerevisiae-based molecular tool kit for manipulation of genes from gram-negative bacteria. Appl Environ Microbiol 72:5027-5036

Singh S, R Chandra, DK Patel, AV Rai (2007). Isolation and characterization of novel Serratia marcescens (AY927692) for pentachlorophenol degradation from pulp and paper mill waste. World J Microb Biotechnol 23:1747-1754

Steel RGD, JH Torrie, DA Dicky (1997). Principles and Procedures of Statistics, A Biometrical Approach, $3^{\text {rd }}$ edn., pp:352-358. McGraw Hill Inc., Book Co., New York, USA

Tran H, A Ficke, T Asiimwe, M Hofte, JM Raaijmakers (2007). Role of the cyclic lipopeptide massetolide A in biological control of Phytophthora infestans and in colonization of tomato plants by Pseudomonas fluorescens. New Phytol 175:731-742

Verhagen BWM, J Glazebrook, T Zhu, HS Chang, LCV Loon, CMJ Pieterse (2004). The transcriptome of rhizobacteria-induced systemic resistance in Arabidopsis. Mol Plant-Microb Interact 17:895-908

Vleesschauwer DV, Y Yang, CV Cruz, M Höfte (2010). Abscisic acidinduced resistance against the brown spot pathogen Cochliobolus miyabeanus in rice involves map kinase-mediated repression of ethylene signaling. Plant Physiol 152:2036-2052

Vleesschauwer DD, P Cornelis, M Höfte (2006). Redox-active pyocyanin secreted by Pseudomonas aeruginosa 7NSK2 triggers systemic resistance to Magnaporthe grisea but enhances Rhizoctonia solani susceptibility in rice. Mol Plant-Microb Interact 19:1406-1419 